\title{
Rentabilidad en maíz (Zea mays L.) y Chile (Capsicum annuum L.) con manejo convencional y alternativo en Autlán, Jalisco
}

\author{
Profitability in maize (Zea mays L.) and chile (Capsicum annuum L.) \\ with conventional and alternative management in Autlán, Jalisco
Oscar Raúl Mancilla Villa ${ }^{*}$, Omar Hernández Vargas ${ }^{1}$, Juan Carlos Manuel Corteéz ${ }^{1}$, José Alfredo Chávez Chávez", Esther Alejandra Castillo Álvarez²,
Rubén Darío Guevara Gutiérrez ${ }^{3}$, José de Jesús Huerta Olague ${ }^{3}$, Álvaro Can Chulim ${ }^{4}$, Héctor Manuel Ortega Escobar ${ }^{5}$, Edgar Iván Sánchez Bernal ${ }^{6}$

\section{RESUMEN}

Los cultivos de maíz y chile jalapeño en México son muy importantes tanto cultural como económicamente. La producción de maíz y chile jalapeño es la principal fuente económica para muchos campesinos del país. Muchos desarrollan agricultura convencional para la producción de sus cultivos. Estas prácticas se basan en la utilización de insumos químicos para la fertilización y control de plagas y, por ende, necesitan fuertes inversiones para poder producir. Algunos agricultores implementan un manejo alternativo de producción, es decir, utilizan abonos orgánicos para mejorar la fertilidad de los suelos de sus campos de cultivo. Además usan algunos productos químicos del mercado para completar la fertilización requerida por los cultivos. Esta investigación se llevó a cabo con recorridos en campos con cultivos de maíz y chile jalapeño en el valle agrícola de Autlán de Navarro, Jalisco, cada uno con dos manejos (alternativo y convencional) y con tres muestreos probabilísticos dirigidos para cada manejo. Se aplicaron los análisis de Valor Actual Neto (VAN), la relación Beneficio/Costo (B/C) y la Tasa Interna de Retorno (TIR), y la mayor productividad y rentabilidad se obtuvo en el manejo alternativo con una mejor relación costo/beneficio y una menor inversión.

Palabras clave: agricultura, beneficio, costo, economía, orgánico.

\begin{abstract}
The corn and chile jalapeño crops in Mexico are essential, culturally, and economically. The production of corn and chile jalapeño is the main economic source for many peasants in the country. Many producers develop conventional agriculture to carry out the production of their crops; these practices are based on the use of chemical inputs for fertilization and pest control. Therefore they need massive investments to be able to produce; in another context, some producers implement an alternative management of production, that is to say, they use organic fertilizers to improve the fertility of the soils of their crop fields and also make use of some chemical products from the market to complete the fertilization required by the crops. In this sense, the present investigation was carried out, with visits to fields with corn and chile crops in the Autlán de Navarro agricultural valley, Jalisco, each with two managements (alternative and conventional) with three probabilistic samplings directed for each handling. The analysis of Net Present Value (NPV), the benefit/cost ratio $(B / C)$, and the Internal Rate of Return (IRR) were implemented. These three parameters were interpreted as obtaining higher productivity and profitability in alternative management with a better relationship cost/ benefit and lower investment.
\end{abstract}

Keywords: agriculture, benefit, cost, economy, organic.

\footnotetext{
Departamento de Producción Agrícola, CUCSUR, Universidad de Guadalajara. Autlán de Navarro, Jalisco, México. Departamento de Contaduría Pública, CUCSUR, Universidad de Guadalajara. Autlán de Navarro, Jalisco, México. Departamento de Ecología y Recursos Naturales, CUCSUR, Universidad de Guadalajara. Autlán de Navarro, Jalisco, México. Universidad Autónoma de Nayarit, Ciudad de la Cultura. Amado Nervo, Tepic, Nayarit. Colegio de Postgraduados, Campus Montecillo. Estado de México. Montecillo, México.

6 Instituto de Ecología, Universidad de la Mar. Puerto Escondido, Oaxaca, México.

* Autor de correspondencia: oscar.mancilla@academicos.udg.mx
}

Fecha de Recepción: 3 de Junio, 2019.

Fecha de Aceptación: 12 de Marzo, 2020. 


\section{INTRODUCCIÓN}

En México el maíz es el cultivo más importante por ser un producto básico en la alimentación de la población. Aunque se cultiva en todas las entidades federativas, las que aportaron $70 \%$ de la producción nacional durante el periodo de 1995 al 2000 fueron Jalisco, Sinaloa, Estado de México, Michoacán, Puebla, Veracruz, Guerrero y Chiapas (SIAP 2010). Algunos autores destacan que el cultivo del chile jalapeño también es fundamental y representativo en la dieta alimenticia de los mexicanos, pues se considera la primera hortaliza domesticada en el continente americano (Vega et al., 2004). La integración de la cultura indígena y europea contribuyó ampliamente en la diversificación de las formas de consumo y el chile jalapeño se produce prácticamente en toda la República Mexicana. Es una de las especies hortícolas más importantes ya que se siembran alrededor de 1,72 millones de hectáreas a nivel mundial. En México se cultiva una gran variedad de chiles y el jalapeño (Capsicum annuиm L.) es uno de los de mayor importancia económica por su amplio consumo, alta rentabilidad y gran demanda de mano de obra (FAO 2008).

Los indicadores para la evaluación económica de proyectos son conceptos valorizados que expresan la rentabilidad de la inversión en una empresa, y sobre esa base se puede tomar la decisión de aceptar o rechazar la realización de un plan, o bien, se evalúa su rentabilidad. También permiten comparar y seleccionar entre diferentes alternativas de inversión (Altuve, 2004). Los indicadores más usados son aquellos que consideran el valor del dinero en el tiempo: el valor actual neto (VAN), la relación beneficiocosto (B/C) y la tasa interna de retorno (TIR). Para evaluar un proyecto de inversión desde el punto de vista económico, el criterio de decisión del VAN es que debe ser igual o mayor que cero. Esto quiere decir que dada una tasa de actualización, el valor presente de los beneficios supera o es igual al valor presente de los costos (Muñante, 2002). En términos generales, el VAN representa la ganancia adicional actualizada que genera el proyecto por encima de la tasa de descuento. Es un indicador financiero que mide los flujos de los ingresos y egresos futuros que tendrá un proyecto, para determinar si luego de descontar la inversión inicial, queda alguna ganancia. El VAN calcula el aumento de la riqueza en el momento actual que resulta equivalente a ejecutar el proyecto $\mathrm{y}$, por lo tanto, cuanto más alto es el VAN, mejor. La técnica consiste en actualizar los costos y beneficios registrados en diferentes periodos y expresarlos en un valor común en un momento determinado. Si el VAN del proyecto es negativo, el proyecto deberá ser rechazado (Mancilla-Villa et al., 2009).

La TIR expresa la tasa de interés real máxima que podría pagar un proyecto por los recursos monetarios utilizados, una vez recuperados los costos de inversión y operación (Muñante, 2002). El criterio formal de selección a través de este indicador es aceptar todos los proyectos independientes cuya TIR sea igual o mayor que la tasa de actualización seleccionada (Altuve, 2004). La TIR representa la tasa de interés más alta que un inversionista podría pagar sin perder dinero, si todos los fondos para el financiamiento de la inversión se tomaran prestados y el capital e interés se pagaran con las entradas en efectivo de la inversión a medida que se fuese produciendo (Mancilla-Villa et al., 2009). Este método considera que una inversión es aconsejable si la TIR resultante es igual o superior a la tasa exigida por el inversor (tasa de descuento), y entre varias alternativas la más convincente será aquella que ofrezca una TIR mayor. Si la TIR es igual a la tasa de descuento, el inversionista es indiferente entre realizar la inversión o no. Si la TIR es menor a la tasa de descuento, el proyecto debe rechazarse (Rocabeth y Padilla, 2009).

La relación beneficio/costo $(\mathrm{B} / \mathrm{C})$ es una herramienta financiera que mide la relación entre los costos y beneficios asociados a un proyecto de inversión con el fin de evaluar su rentabilidad. Se entiende el proyecto de inversión no solo como la creación de un nuevo negocio, sino también como inversiones que se pueden hacer en un negocio en marcha, tales como el desarrollo de nuevos productos o la adquisición de nueva maquinaria para infraestructura (Vijaya, 2014). De acuerdo con el criterio formal de selección de los proyectos de inversión basados en este indicador, se acepta el proyecto o se cataloga como rentable si la $\mathrm{B} / \mathrm{C}$ es mayor que uno (Muñante, 2002). El objetivo de este trabajo es estimar la rentabilidad económica con base en los rendimientos en parcelas agrícolas bajo la siembra de maíz y chile jalapeño, con prácticas de fertilización alternativa y convencional. 


\section{Materiales y Métodos}

\section{Área de estudio}

En esta investigación se consideraron tanto los costos como los ingresos generados en la producción de maíz y chile jalapeño bajo dos tipos de fertilización en el valle Autlán de Navarro-El Grullo, que se localiza al suroeste del estado de Jalisco, México (Figura 1), entre las coordenadas extremas de $19^{\circ} 35^{\prime} 30^{\prime \prime}$ a los $19^{\circ} 54^{\prime} 45^{\prime \prime}$ norte y $104^{\circ} 07^{\prime} 00^{\prime \prime}$ a $104^{\circ} 29^{\prime} 35^{\prime \prime}$ oeste, con una altitud de 900 m (INEGI 2000).

\section{Diseño de muestreo}

Se establecieron los cultivos de maíz y chile jalapeño por separado con una superficie de una hectárea cada uno. El tiempo de establecimiento de los cultivos fue de acuerdo al ciclo de vida de cada hortaliza, con dos tratamientos. El primer tratamiento consistió en el manejo convencional o químico en el suelo, el cual suministra los nutrientes en forma química o sintética que necesitan las plantas en el momento, cantidad y forma adecuada (Bellapart, 1988). El segundo tratamiento es el manejo del suelo alternativo o complementario, que consiste en la combinación de fertilizantes sintéticos o químicos con fertilizantes orgánicos derivados de residuos de animales y vegetales, para proporcionar nutrientes de una manera complementaria (Cásseres, 1980). Se realizaron tres muestreos probabilísticos dirigidos en cada tipo de manejo, en cultivos de maíz y chile jalapeño, con dos sistemas de manejo, el alternativo y el convencional, para ambos (Tabla 1), en los meses de septiembre a noviembre de 2018, al inicio, desarrollo y fructificación de los cultivos, con intervalos de 30 días cada muestreo (Vivanco, 2005). El método usado para cálculo de los indicadores financieros fue el de aproximaciones sucesivas propuestas por Barrios y Portillo (1989).

Mediante los tres muestreos se determinó la fertilidad del suelo bajo los dos tipos de manejo, el alternativo y el convencional, correspondiendo a una hectárea cada uno. Para esto se colectaron
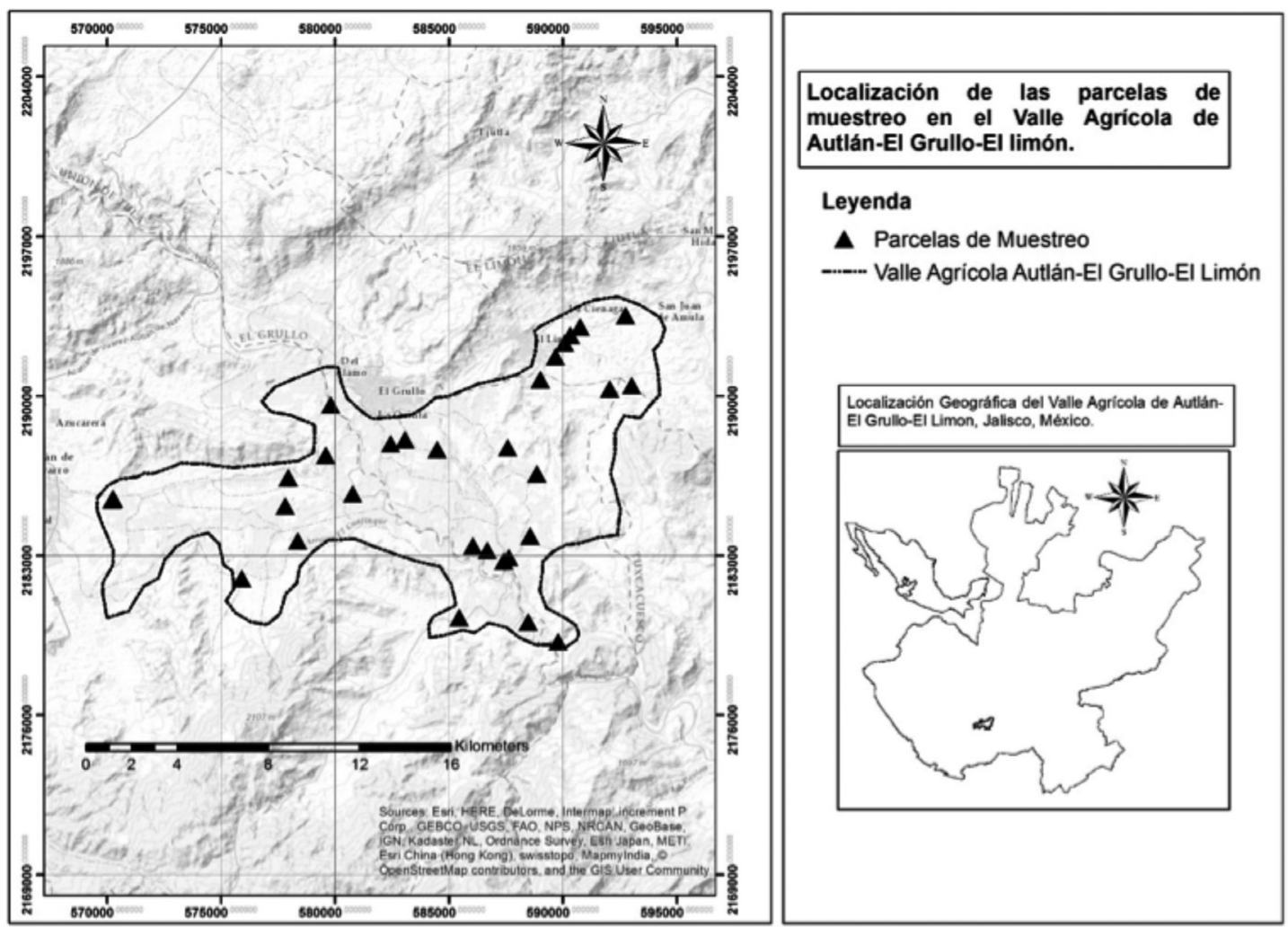

Figura 1. Localización geográfica del valle agrícola de Autlán. 
tres muestras de suelo en los cultivos de maíz y chile jalapeño, en la etapa inicial, de desarrollo y fructificación, a fin de precisar la demanda de nutrientes $(\mathrm{N}, \mathrm{P}, \mathrm{K}, \mathrm{Ca}$ y $\mathrm{Mg}$ ) y los parámetros ( $\mathrm{pH}, \mathrm{Ce}, \mathrm{CIC}$ y MO) que presenta el suelo en cada etapa del cultivo. Con esto se realizó parte del análisis sobre los costos para determinar la relación costo/beneficio. Posteriormente la estimación de la rentabilidad económica se realizó mediante la definición de los costos de producción, el precio de venta que representan los ingresos y la diferencia entre estos dos. Los costos considerados son aquellos que van desde la instalación de la huerta, el costo de los insumos utilizados tanto químicos como orgánicos (Tabla 1), hasta los necesarios para que funcione cada periodo productivo del cultivo. Los beneficios que se tomaron en cuenta en el estudio son los obtenidos por la venta del maíz para elote, considerando el precio fijo que se determinó al establecer el cultivo, y de acuerdo al contrato suscrito en el cual se señala el compromiso del comprador hacia el productor de adquirir el producto a un precio establecido. Es importante mencionar que los precios de mercado del elote son idénticos para ambos manejos.

\section{Parámetros de rentabilidad}

Los parámetros de rentabilidad TIR, VAN, C/B, (Tobar 2005) se desarrollaron al final del ciclo cuando se tuvo conocimiento de la inversión inicial y producción total en cada uno de los manejos, es decir, los costos e ingresos de producción. La valoración económica se realizó tomando la metodología empleada en los trabajos de la IFA (2002). Los principales indicadores económicos evaluados fueron el beneficio neto y el efecto económico. El VAN se calcula mediante la ecuación siguiente:

$$
V A N=t=1 T B t-C t(1-i) t
$$

\section{Dónde:}

VAN = valor actual neto $(\$) \mathrm{Bt}=$ Beneficio de cada año (\$) $\mathrm{Ct}=$ Costos de cada año (\$) $\mathrm{i}=$ Tasa de interés $(\%) \mathrm{T}=$ Número de periodos de análisis o de vida útil del proyecto $t=$ Cada periodo del proyecto (año $1,2, \ldots ., \mathrm{T}$ ).

A continuación se presenta la expresión matemática formal de la TIR, ecuación, la cual representa una tasa de actualización que iguala el VAN a cero (Sánchez y Salazar, 1994).

$$
T I R=t=1 T B t-C t(1+i) t=0
$$

Dónde:

TIR $=$ Tasa interna de retorno $(\%) \mathrm{Bt}=$ Beneficios de cada año (\$) $\mathrm{Ct}=$ Costos de cada año (\$). $\mathrm{i}=$ Tasa de interés $(\%) \mathrm{T}=$ Número de periodos de análisis o de vida útil del proyecto $\mathrm{t}=$ cada periodo del proyecto $($ año $1,2 \ldots \mathrm{T})(1+i)$ $-1=$ Factor de actualización.

\begin{tabular}{|c|c|c|c|c|c|c|c|}
\hline Cultivo & Manejo & $\begin{array}{l}\text { Fertilizantes } \\
\text { químicos }\end{array}$ & $\begin{array}{l}\text { Fertilizantes } \\
\text { orgánicos }\end{array}$ & Cultivo & Manejo & $\begin{array}{l}\text { Fertilizantes } \\
\text { químicos }\end{array}$ & $\begin{array}{c}\text { Fertilizantes } \\
\text { orgánicos }\end{array}$ \\
\hline \multirow{10}{*}{ Maíz } & \multirow{6}{*}{ Convencional } & Urea & & \multirow{10}{*}{$\begin{array}{l}\text { Chile } \\
\text { jalapeño }\end{array}$} & \multirow{6}{*}{ Convencional } & \multirow{6}{*}{$\begin{array}{l}\text { Nitrato de calcio } \\
\text { Nitrato de magnesio } \\
\text { Nitrato de potasio } \\
\text { Cloruro de potasio } \\
\text { Fosfato diamónico } \\
\text { Fosfonitrato }\end{array}$} & \\
\hline & & Fosfonitrato & & & & & \\
\hline & & Nitrato de potasio & & & & & \\
\hline & & Fosfato diamónico & & & & & \\
\hline & & Urea & $\overline{\text { Ácidos húmicos y }}$ & & & & \\
\hline & & Fosfonitrato & $\begin{array}{l}\text { fulvicos.Humus de } \\
\text { lombriz y guano }\end{array}$ & & & & \\
\hline & & & & & \multirow{4}{*}{ Alternativo } & Nitrato de calcio & $\begin{array}{l}\text { Ácidos húmicos y } \\
\text { fulvicos }\end{array}$ \\
\hline & & & & & & Nitrato de magnesio & $\begin{array}{l}\text { Humus de lombriz } \\
\text { y guano }\end{array}$ \\
\hline & & & & & & Nitrato de potasio & Micorrizas \\
\hline & & & & & & Cloruro de potasio & Algas marinas \\
\hline
\end{tabular}

Tabla 1. Fertilizantes utilizados para los dos tipos de manejo en los cultivos de maíz y chile jalapeño. 
La relación $\mathrm{B} / \mathrm{C}$ es una forma práctica y útil en la determinación de la viabilidad de un proyecto y si este es recomendable o no, donde según Mancilla-Villa et al., 2009, la regla de operación es la siguiente:

Si B/C > 1, la obra es costeable. Si B/C $<1$, la obra es incosteable. $\mathrm{Si} \mathrm{B} / \mathrm{C}=1$, existe un indecisión.

Según Mancilla-Villa et al. (2009) la relación beneficio/costo se calcula mediante la ecuación.

$$
B C t-1 T B j(1+i) n t-1 T C j(1+i) j
$$

Dónde: $\mathrm{B} j=$ beneficio de cada año (\$) $\mathrm{C} j=$ costo de cada año $(\$) \mathrm{i}=$ tasa de interés (\%) n= número de periodos de análisis o de vida útil del proyecto $\mathrm{j}=$ cada periodo del proyecto (año $1,2 \ldots$. T).

\section{Resultados y discusión}

Se utilizaron dos tipos de manejo para el cultivo de maíz con la finalidad de obtener la rentabilidad de cada sistema durante cinco temporadas diferentes. Al analizar los costos e ingresos que se generaron en ambos manejos para los cultivos correspondientes, en cuanto a los flujos de efectivos, los ingresos y egresos actualizados, se obtuvieron los resultados que se muestran en las Tablas 2 y 3 . Por otra parte, se evaluaron los flujos de efectivos correspondientes a las cinco temporadas en el cultivo de chile jalapeño, bajo los manejos alternativos y convencionales. Se presentan en las Tablas 4 y 5. El VAN, TIR y B/C son los indicadores de rentabilidad, que permiten tomar la decisión de llevar a cabo el proyecto o no. Se presentan los valores calculados para los indicadores en maíz con ambos manejos (Tabla 6). $\mathrm{El}$ valor de relación $\mathrm{B} / \mathrm{C}$ indica que durante las cinco temporadas de evaluación con una tasa de interés de $10 \%$ anual, se tiene que, por cada peso invertido en el manejo alternativo de maíz se obtendrán $\$ 8,22$ pesos; para el VAN se logrará una utilidad neta de $\$ 1.161 .346,40$ durante el tiempo evaluado y para la TIR se tendrá una tasa de $347,23 \%$. Se presenta la relación costo/beneficio en la cual se puede observar que la diferencia es considerable (Figura 2), ya que el valor mínimo es de 1,18 en el cultivo de trigo y el valor máximo 8,22

Tabla 2. Costos, beneficios y flujo para las cinco temporadas de evaluación para el manejo alternativo de maíz.

\begin{tabular}{lccccccr}
\hline Temporada & Ingresos & Costos & $\begin{array}{c}\text { Flujo de } \\
\text { Efectivo }\end{array}$ & $\begin{array}{c}\text { Tasa } \\
(1+\mathrm{t})-\mathrm{n}\end{array}$ & $\begin{array}{c}\text { Ingresos } \\
\text { Actualizados }\end{array}$ & $\begin{array}{c}\text { Egresos } \\
\text { Actualizados }\end{array}$ & $\begin{array}{c}\text { Flujo de } \\
\text { Efectivo } \\
\text { Actualizado }\end{array}$ \\
\hline Temporada 0 & $\$-$ & $\$ 30.000,00$ & $\$(30.000,00)$ & 1 & $\$-$ & $\$ 30.000,00$ & $\$(30.000,00)$ \\
Temporada 1 & $\$ 66.000,00$ & $\$ 31.500,00$ & $\$ 34.500,00$ & 0.90909091 & $\$ 120.000,00$ & $\$ 28.636,36$ & $\$ 91.363,64$ \\
Temporada 2 & $\$ 132.000,00$ & $\$ 33.075,00$ & $\$ 98.925,00$ & 0.82644628 & $\$ 163.636,36$ & $\$ 27.334,71$ & $\$ 136.301,65$ \\
Temporada 3 & $\$ 198,000,00$ & $\$ 34.728,75$ & $\$ 163.271,25$ & 0.7513148 & $\$ 198.347,11$ & $\$ 26.092,22$ & $\$ 172.254,88$ \\
Temporada 4 & $\$ 264.000,00$ & $\$ 36.465,19$ & $\$ 227.534,81$ & 0.68301346 & $\$ 225.394,44$ & $\$ 24.906,21$ & $\$ 200.488,23$ \\
Temporada 5 & $\$ 330.000,00$ & $\$ 38.288,45$ & $\$ 291.711,55$ & 0.62092132 & $\$ 614.712,11$ & $\$ 23.774,11$ & $\$ 590.938,00$ \\
Total & $\$ 990.000,00$ & $\$ 204.057,38$ & $\$ 785.942,62$ & - & $\$ 1.322 .090,02$ & $\$ 160.743,63$ & $\$ 1.161 .346,40$ \\
\hline
\end{tabular}

Tabla 3. Costos, beneficios y flujo para las cinco temporadas de evaluación para el manejo convencional de maíz.

\begin{tabular}{lccccccr}
\hline Temporada & Ingresos & Costos & $\begin{array}{c}\text { Flujo de } \\
\text { Efectivo }\end{array}$ & $\begin{array}{c}\text { Tasa } \\
(1+\mathrm{t})-\mathrm{n}\end{array}$ & $\begin{array}{c}\text { Ingresos } \\
\text { Actualizados }\end{array}$ & $\begin{array}{c}\text { Egresos } \\
\text { Actualizados }\end{array}$ & $\begin{array}{c}\text { Flujo de } \\
\text { Efectivo } \\
\text { Actualizado }\end{array}$ \\
\hline Temporada 0 & $\$-$ & $\$ 28.060,00$ & $\$(28.060,00)$ & 1.00000 & $\$-$ & $\$ 28.060,00$ & $\$(28.060,00)$ \\
Temporada 1 & $\$ 54.000,00$ & $\$ 29.463,00$ & $\$ 24.537,00$ & 0.90909091 & $\$ 49.090,91$ & $\$ 26.784,55$ & $\$ 22.306,36$ \\
Temporada 2 & $\$ 108.000,00$ & $\$ 30.936,15$ & $\$ 77.063,85$ & 0.82644628 & $\$ 89.256,20$ & $\$ 25.567,07$ & $\$ 63.689,13$ \\
Temporada 3 & $\$ 162.000,00$ & $\$ 32.482,96$ & $\$ 129.517,04$ & 0.7513148 & $\$ 121.713,00$ & $\$ 24.404,93$ & $\$ 97.308,07$ \\
Temporada 4 & $\$ 216.000,00$ & $\$ 34.107,11$ & $\$ 181.892,89$ & 0.68301346 & $\$ 147.530,91$ & $\$ 23.295,61$ & $\$ 124.235,29$ \\
Temporada 5 & $\$ 270.000,00$ & $\$ 35.812,46$ & $\$ 234.187,54$ & 0.62092132 & $\$ 167.648,76$ & $\$ 22.236,72$ & $\$ 145.412,04$ \\
Total & $\$ 810.000,00$ & $\$ 190.861,67$ & $\$ 619.138,33$ & & $\$ 575.239,77$ & $\$ 150.348,87$ & $\$ 424.890,90$ \\
\hline
\end{tabular}


Tabla 4. Costos, beneficios y flujo para las cinco temporadas de evaluación para el manejo alternativo de chile.

\begin{tabular}{lccccccc}
\hline Temporada & Ingresos & Costos & $\begin{array}{c}\text { Flujo de } \\
\text { efectivo }\end{array}$ & $\begin{array}{c}\text { Tasa } \\
(1+\mathrm{t})-\mathrm{n}\end{array}$ & $\begin{array}{c}\text { Ingresos } \\
\text { actualizados }\end{array}$ & $\begin{array}{c}\text { Egresos } \\
\text { actualizados }\end{array}$ & $\begin{array}{c}\text { Flujo de } \\
\text { efectivo } \\
\text { actualizado }\end{array}$ \\
\hline Temporada 0 & $\$-$ & $\$ 174.425,00$ & $\$(174.425,00)$ & 1 & $\$-$ & $\$ 174.425,00$ & $\$(174.425,00)$ \\
Temporada 1 & $\$ 280.000,00$ & $\$ 183.146,25$ & $\$ 96.853,75$ & 0.89285714 & $\$ 250.000,00$ & $\$ 163.523,44$ & $\$ 86.476,56$ \\
Temporada 2 & $\$ 560.000,00$ & $\$ 192.303,56$ & $\$ 367.696,44$ & 0.79719388 & $\$ 446.428,57$ & $\$ 153.303,22$ & $\$ 293.125,35$ \\
Temporada 3 & $\$ 840.000,00$ & $\$ 201.918,74$ & $\$ 638.081,26$ & 0.71178025 & $\$ 597.895,41$ & $\$ 143.721,77$ & $\$ 454.173,64$ \\
Temporada 4 & $\$ 1.120 .000,00$ & $\$ 212.014,68$ & $\$ 907.985,32$ & 0.63551808 & $\$ 711.780,25$ & $\$ 134.739,16$ & $\$ 577.041,09$ \\
Temporada 5 & $\$ 1.400 .000,00$ & $\$ 222.615,41$ & $\$ 1.177 .384,59$ & 0.56742686 & $\$ 794.397,60$ & $\$ 126.317,96$ & $\$ 668.079,64$ \\
Total & $\$ 4.200 .000,00$ & $\$ 1.186 .423,64$ & $\$ 3.013 .576,36$ & & $\$ 2.800 .501,83$ & $\$ 896.030,55$ & $\$ 1.904 .471,27$ \\
\hline
\end{tabular}

Tabla 5. Costos, beneficios y flujo para las cinco temporadas de evaluación para el manejo convencional de chile.

\begin{tabular}{lccccccc}
\hline Temporada & Ingresos & Costos & $\begin{array}{c}\text { Flujo de } \\
\text { Efectivo }\end{array}$ & $\begin{array}{c}\text { Tasa } \\
(1+\mathrm{T})-\mathrm{N}\end{array}$ & $\begin{array}{c}\text { Ingresos } \\
\text { actualizados }\end{array}$ & $\begin{array}{c}\text { Egresos } \\
\text { actualizados }\end{array}$ & $\begin{array}{c}\text { Flujo de efectivo } \\
\text { actualizado }\end{array}$ \\
\hline Temporada 0 & $\$-$ & $\$ 168.425,00$ & $\$(168.425,00)$ & 1 & $\$-$ & $\$ 168.425,00$ & $\$(168.425,00)$ \\
Temporada 1 & $\$ 240.000,00$ & $\$ 176.846,25$ & $\$ 63.153,75$ & 0,89285714 & $\$ 214.285,71$ & $\$ 157.898,44$ & $\$ 56.387,28$ \\
Temporada 2 & $\$ 480.000,00$ & $\$ 185.688,56$ & $\$ 294.311,44$ & 0,79719388 & $\$ 382.653,06$ & $\$ 148.029,79$ & $\$ 234.623,28$ \\
Temporada 3 & $\$ 720.000,00$ & $\$ 194.972,99$ & $\$ 525.027,01$ & 0,71178025 & $\$ 512.481,78$ & $\$ 138.777,92$ & $\$ 373.703,85$ \\
Temporada 4 & $\$ 960.000,00$ & $\$ 204.721,64$ & $\$ 755.278,36$ & 0,63551808 & $\$ 610.097,36$ & $\$ 130.104,30$ & $\$ 479.993,05$ \\
Temporada 5 & $\$ 1.200 .000,00$ & $\$ 214.957,72$ & $\$ 985.042,28$ & 0,56742686 & $\$ 680.912,23$ & $\$ 121.972,78$ & $\$ 558.939,44$ \\
Total & $\$ 3.600 .000,00$ & $\$ 1.145 .612,17$ & $\$ 2.454 .387,83$ & - & $\$ 2.400 .430,14$ & $\$ 865.208,23$ & $\$ 1.535 .221,90$ \\
\hline
\end{tabular}

Tabla 6. Comparación de los indicadores de rentabilidad del maíz bajo el manejo convencional y alternativo.

\begin{tabular}{|c|c|c|}
\hline \multicolumn{3}{|c|}{ Indicadores de rentabilidad del maíz } \\
\hline & Alternativo & Convencional \\
\hline VAN & $\$ 1.161 .346,40$ & $\$ 424.890,90$ \\
\hline TIR & $347,23 \%$ & $157,57 \%$ \\
\hline $\mathrm{B} / \mathrm{C}$ & 8,22 & 3,83 \\
\hline
\end{tabular}

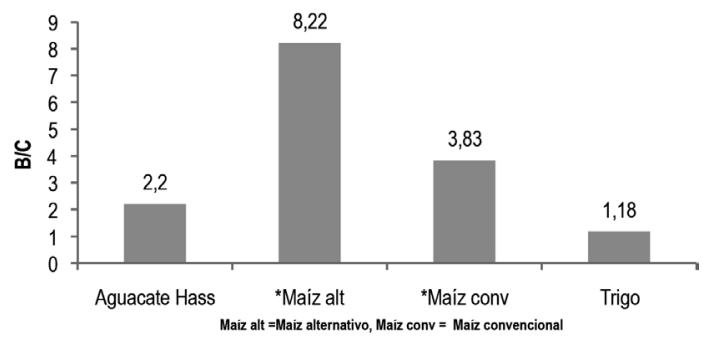

Figura 2. Comparación de Valores Costo/Beneficio aguacate Hass, trigo, maíz bajo el manejo alternativo y convencional.

en el cultivo de maíz bajo el manejo alternativo, resultando una diferencia de 7,04. Esto significa que aunque el trigo y el aguacate Hass son cultivos muy populares a nivel mundial, el maíz cultivado bajo condiciones alternativas resulta más rentable para el agricultor y, por ende, le genera mayores ganancias. Con respecto al cultivo de chile jalapeño, se presentan los indicadores de rentabilidad en ambos manejos, lo que permite determinar cuál de los dos resulta con mayor rentabilidad (Tabla 7). La relación $\mathrm{B} / \mathrm{C}$ indica que durante las cinco temporadas de evaluación bajo el manejo convencional con la tasa de interés considerada, por cada peso invertido se obtendrán $\$ 2,13$ pesos de ganancia. Con base en la estimación del VAN se observa una utilidad neta de \$1.904.471,27 durante el tiempo evaluado. Para la TIR se obtiene

Tabla 7. Comparación de los indicadores de rentabilidad del chile bajo el manejo convencional y alternativo.

\begin{tabular}{lcc}
\hline \multicolumn{3}{c}{ Indicadores de rentabilidad del chile } \\
\hline \multicolumn{2}{c}{ Alternativo } & Convencional \\
\hline VAN & $\$ 1.904 .471,27$ & $\$ 1.535 .221,90$ \\
TIR & $122,85 \%$ & $105,41 \%$ \\
B/C & 3,13 & 2,77 \\
\hline
\end{tabular}




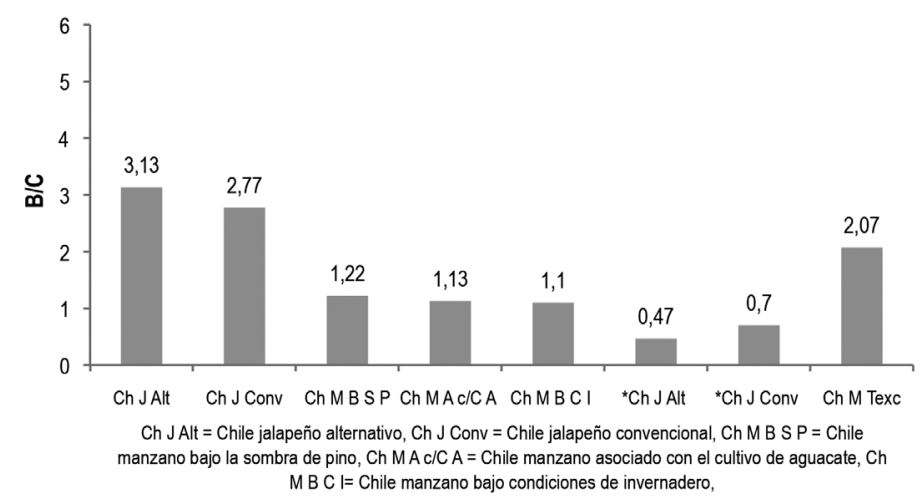

Figura 3. Comparación de Valores Costo/Beneficio de diferentes tipos de chile en condiciones diferentes a las presentadas en este estudio.

una tasa de $122 \%$. Los indicadores son afectados por la inflación del 5\% anual.

Al finalizar las cinco temporadas del cultivo de maíz bajo el manejo alternativo se obtuvieron ingresos totales actualizados de \$1.322.090,02 con egresos totales actualizados de $\$ 160.743,63$ y un flujo de efectivo actualizado de $\$ 1.161 .346,40$. El flujo de efectivo es la diferencia entre los ingresos y costos, y los ingresos y egresos actualizados es lo que se obtiene con una tasa de interés de $10 \%$. Los beneficios del uso de abonos orgánicos son muy amplios, ya que además de aportar materia orgánica humificada y nutrimentos al suelo, se ha demostrado que pueden prevenir, controlar e influir en la severidad del ataque de patógenos del suelo (Pedroza y Samaniego, 2003). Además diversos autores aseguran que si se invierte en el manejo alternativo como una opción para cultivar, el agricultor puede obtener mayores beneficios económicos, pues como se muestra en el cuadro anterior, los ingresos totales actualizados son ocho veces mayores, en comparación con los egresos totales actualizados. Resulta una gran decisión adoptar este tipo de manejos en los cultivos de maíz. Al finalizar todas las temporadas del cultivo de maíz bajo el manejo convencional se obtuvieron ingresos totales actualizados de $\$ 575.239,77$ con egresos totales actualizados de $\$ 150.348,87$ y un flujo de efectivo actualizado de $\$ 424.890,90$. Después de analizar los flujos de efectivo actualizados se puede observar que los ingresos son tres veces mayores que los egresos al final de las cinco temporadas de cultivo convencional. Existen publicaciones donde se concluye que los sistemas de producción intensivos tienen ventajas no sólo en la calidad y cantidad de producto cosechado, sino también en la oportunidad de su producción (Pérez et al., 1997; Pérez et al., 2004).

Para el cultivo de chile jalapeño bajo el manejo alternativo se obtuvieron ingresos totales actualizados de $\$ 2.800 .501,83$ con egresos totales actualizados de $\$ 896.030,55$ y un flujo de efectivo actualizado de $\$ 1.904 .471,27$. Como se puede observar en la Tabla 4, los ingresos totales actualizados son tres veces mayores a los egresos totales actualizados, lo que resulta muy favorable para el productor, pues significa que obtendrá ingresos superiores a la inversión inicial. El manejo orgánico ofrece la posibilidad de competir en la comercialización del chile jalapeño $\mathrm{y}$, adicionalmente, incrementa la fertilidad del suelo (Seufert et al., 2012).

Al concluir las cinco temporadas del cultivo de chile jalapeño bajo el manejo convencional se obtuvieron ingresos totales actualizados de $\$ 2.400 .430,14$ con egresos totales actualizados de $\$ 865.208,23$ y un flujo de efectivo actualizado de $\$ 1.535 .221,90$. Respecto al cultivo convencional, los ingresos totales actualizados son dos veces superiores a los egresos totales actualizados, y en comparación con el manejo alternativo la diferencia es mayor, por lo que resulta más favorable optar por el otro manejo. Existen resultados de investigaciones de autores como Jaik-Dipp y Tena-Flores (1990), Cai y Corke (1999), según los cuales los costos de producción para los productores son muy altos y ocasionan que las ganancias sean menores. A pesar de esto cultivan de manera tradicional en la zona (Enama, 1994; Judd, 2008; Sánchez, 1980; Sánchez et al., 1991).

De acuerdo con los indicadores estimados, se establece que del valor de la relación $\mathrm{B} / \mathrm{C}$ por cada 
peso invertido en el manejo convencional de maíz se obtendrán $\$ 2,83$ pesos de ganancia durante las cinco temporadas evaluadas: Es decir, se tiene una ganancia mayor de $\$ 4,39$ pesos en el manejo alternativo al compararse con el convencional. Para el VAN se obtendrá una utilidad neta de $\$ 424.890,90$, lo que significa una utilidad mayor de $\$ 436.455,50$ del manejo alternativo respecto al convencional, mientras que para la TIR será de $157,57 \%$. En este valor resalta una diferencia del manejo alternativo de $189,66 \%$ respecto al manejo convencional. Cabe señalar que los indicadores son afectados por la inflación de 5\% anual. Con el propósito de abordar la rentabilidad que reportan otros autores con investigaciones en diferentes cultivos, se describen a continuación dos casos. Peña Urquiza et al. (2015), en el Estado de México, obtuvieron los siguientes resultados en aguacate Hass: un VAN de $\$ 15368,80$, una TIR de $41,9 \%$ y un B/C de 2,2, los cuales son diferentes a los registrados en este trabajo. Respecto al maíz se encontró que en el manejo alternativo el valor del costo/ beneficio es superior seis veces a lo obtenido por Peña Urquiza et al. (2015). Con esto queda comprobado que a pesar de que el aguacate es un cultivo muy usual y rentable, existen otras condiciones de cultivos de los cuales se pueden lograr beneficios altos, como por ejemplo el maíz. Esto se debe a que la inversión es menor tanto en materia prima como en mano de obra y gastos indirectos, lo que contribuye a obtener ganancias mayores, así como volúmenes más altos de producción.

En un estudio de rentabilidad de trigo en la Costa de Hermosillo, Sonora, Retes López et al. (2013) observaron que la Relación Beneficio-Costo fue de 1,18 , es decir, que por cada peso que el productor invierta, recupera 18 centavos, lo cual es aceptable al estar esta relación por arriba del mínimo aceptado de 1 o mayor de 1 . Los resultados son diferentes a los reportados en este trabajo, puesto que con el manejo alternativo se obtuvo un VAN de \$1.161.346,40, una TIR de 347,23\% y un $\mathrm{B} / \mathrm{C} 8,22$ y con un manejo convencional un VAN \$ 424.890,90, una TIR $157,57 \%$ y un B/C 3,83 . Esto se debe a que los cultivos son diferentes. Otra situación que influyó fue el tipo de tierra y las condiciones climatológicas. Sin embargo, el factor más importante fue la inversión hecha en cada cultivo. En este trabajo tanto los ingresos actualizados como los egresos actualizados son muy altos y en consecuencia la relación costo/beneficio también es considerable en referencia a los estudios de aguacate Hass y trigo antes mencionados. El valor de $\mathrm{B} / \mathrm{C}$ durante las cinco temporadas de evaluación bajo el manejo alternativo en cultivo de chile jalapeño, con una tasa de interés de $12 \%$ anual, representó que por cada peso invertido se obtendrán $\$ 1,77$ pesos de ganancia con una diferencia de 0,36 pesos entre el cultivo alternativo y el convencional. Para el VAN la utilidad neta generada se ubicó en $\$ 1.535 .221,90$ y la diferencia fue de $\$ 369.249,37$ entre ambos cultivos quedando con mayor utilidad el alternativo. La TIR fue de $105,41 \%$ con una diferencia de $17,44 \%$ y de nuevo el manejo alternativo es superior al convencional. Estos indicadores son afectados por la inflación del 5\% anual. Los resultados vuelven a favorecer al manejo alternativo como mejor opción de cultivo en la región del valle de Autlán de Navarro en la cosecha de chile jalapeño. En un estudio de rentabilidad de chile manzano en México bajo tres diferentes sistemas de producción se encontraron los siguientes resultados: con el sistema a campo abierto bajo la sombra de pino se obtuvo un VAN $\$ 23.975,86$, una TIR de $30,08 \%$ y una B/C 1,22 ; con el sistema a campo abierto asociado con el cultivo de aguacate se obtuvo un VAN $\$ 12.546,15$, una TIR de $22,65 \%$ y una B/C 1,13; con el sistema intensivo bajo condiciones de invernadero $\left(1,500 \mathrm{~m}^{2}\right)$ se obtuvo un VAN \$60.661,87, una TIR de 21,41\% y una B/C 1,10 (Espinosa-Torres et al., 2016). En un estudio hecho en el cultivo de chile jalapeño por Morón y Alayón (2014) se encontraron los siguientes resultados: en un área de $1,802 \mathrm{~m}^{2}$ con manejo convencional y con un costo por hectárea de $\$ 122.300,00$ se obtuvo un costo/beneficio de 0,70 , mientras que para el manejo alternativo en un área de $4,400 \mathrm{~m}^{2}$ con un costo por hectárea de \$ 45.800,00 se reportó un costo/beneficio de 0,47 . En otro estudio hecho en cultivos de chile manzano en Texcoco (Espinosa-Torres et al., 2016) se obtuvo un VAN de \$ 799.594,88, en tanto que la tasa interna de rentabilidad (TIR) fue de $45,5 \%$, lo cual quiere decir que es la máxima tasa de descuento que puede soportar el proyecto. $\mathrm{La}$ relación beneficio-costo $(\mathrm{RB} / \mathrm{C})$ fue de 2,07 , lo cual indica que por cada peso invertido se ganarán $\$ 1,07$ durante la vida útil del proyecto, con una inversión total de $\$ 237.371,83$ en el primer año.

Estos resultados difieren de los observados en este estudio en cultivo de chile jalapeño, donde el 
valor de VAN fue de \$1.904 471,27, mucho mayor a los presentados anteriormente. Con una TIR de $122,85 \%$ este valor es superior a cualquiera de los mencionados respecto a los diferentes tipos de chile y B/C 3.13 bajo el sistema de cultivo alternativo, y es la más alta relación obtenida en comparación con las siete registradas en los otros estudios realizados. Un VAN de \$1.535.221,90, una TIR de $105,41 \%$ y una B/C de 2,77 bajo el sistema de cultivo convencional. En este sentido se tiene que por cada peso de costo total invertido en el proyecto, se logran 3,13 pesos de beneficios totales con el manejo alternativo y 2,77 pesos con el manejo convencional. Asimismo, la adición de abonos orgánicos ayuda en la disminución de los costos de producción del cultivo, según Willer y Yussefi (2000), lo que permite obtener mayores utilidades. Se muestra una comparación de los diferentes valores registrados en ocho estudios realizados en chiles en distintos lugares del país incluyendo los de este trabajo (Figura 3). Allí se puede observar con cuál cultivo se logra mayor costo/beneficio, y con esta información se pueden tomar decisiones respecto a cuál es más viable y se obtendrán mayores ganancias. Como se muestra en la Figura 3 el valor máximo es de 3,13 encontrado en este estudio y el valor mínimo 0,47 obtenido por Morón y Alayón (2014) considerando que ambos son chiles jalapeños bajo un manejo alternativo. Resulta una diferencia de 2,66 en la relación costo/beneficio, y es superior el valor reportado en este trabajo. Son muchos los factores que pudieron influir para llegar a estos resultados, por ejemplo, la inversión realizada por cada productor y las condiciones ambientales como el clima, los nutrientes del suelo y la calidad del agua. Cabe destacar que la diferencia resultante entre ambos manejos es bastante aceptable, por lo que se puede concluir que las condiciones para cosechar este tipo de hortalizas son muy buenas en el valle de Autlán de Navarro, pues los beneficios que se obtienen favorecen tanto a la tierra como a la economía del productor.

\section{Conclusión}

De acuerdo con la relación costo/beneficio encontrada en este trabajo en los cultivos de maíz, se determinó que el manejo alternativo es 4,39 veces superior al convencional. Respecto al valor VAN resultó una diferencia de 436.455,50 de utilidad comparado con el manejo convencional y para TIR hubo una diferencia de $189,66 \%$. Así queda comprobado que el manejo alternativo es más rentable y genera mayor utilidad en este tipo de cultivos. Mientras que en el cultivo de chile jalapeño la diferencia en el costo/beneficio fue de 0,36 pesos y es superior el manejo alternativo. La VAN tuvo una diferencia de 369.249,37 de utilidad en el manejo alternativo. Respecto al valor TIR se encontró $17,44 \%$ por encima el manejo alternativo. Así se vuelve a demostrar que para este tipo de cultivos y en las condiciones en las que se trabajó, es mejor el manejo alternativo, pues además de ayudar a los suelos, los volúmenes de producción son muy altos y las ganancias resultan considerables. Adicionalmente, una opción potencial para el productor es el manejo alternativo en sus cultivos puesto que estos generan mayor aprovechamiento de los recursos naturales. Por ello se recomienda en la medida de lo posible apoyar su desarrollo, para que esto se traduzca en una mayor rentabilidad y así garantizar su continuidad. La elección del manejo alternativo resultaría más favorable tanto para el productor como para el terreno donde se implemente. En este sentido el manejo alternativo presenta mayores ganancias y, por ende, mayor rentabilidad a corto plazo, la fertilidad del suelo se mantiene y además a mediano y largo plazo se generan importantes reducciones en los costos de producción, aunado al incremento en los rendimientos. Otro aspecto importante a considerar en futuras investigaciones es el sobreprecio de productos sanos obtenidos de manera sustentable. Ello permitiría la obtención de mayores ganancias para los productores, además de inclinar la balanza a favor del manejo alternativo como forma sostenible de producción de alimentos coadyuvando a la soberanía alimentaria y la salud humana. 


\section{Literatura Citada}

Altuve, J.G.

2004. El uso del valor actual neto y la tasa interna de retorno para la valoración de las decisiones de inversión. Actualidad Contable Faces Universidad de los Andes. 7: 7-17.

Barrios-Puente, G.M.; Portillo-V.

1989. Elaboración y evaluación de proyectos de inversión en el sector forestal. Revista Agrosociedad. 1: 19-37.

Bellapart, C.

1988. Agricultura biológica en equilibrio con la agricultura química: fertilización natural, la agricultura del futuro. AEDOS, Barcelona, España. 280 p.

Cai, Y.; Corke H.

1999. Amaranthus betacyanin pigments applied in model food sys tems. Journal of Food Science, 64: 869-873.

Cásseres, E.

1980. Producción de hortalizas. Bib Ortón IICA/CATIE, $400 \mathrm{p}$.

Enama, M.

1994. Culture: the missing nexus in ecological economics perspective. Ecological Economics, 10: 93-95.

Espinosa, T.L.E.; Ramírez, A.O.

2016. Rentabilidad de chile manzano (Capsicum pubescens $\mathrm{R}$ y $\mathrm{P}$ ) producido en invernadero en Texcoco, Estado de México. Revista Mexicana de Ciencias Agrícolas. 7(2): 325-335.

FAO-Centro de Comercio Internacional

2001. Los mercados mundiales de frutas y verduras orgánicas: oportunidades para los países en desarrollo en cuanto a la producción y exportación de productos hortícolas orgánicos. Roma, Italia.

Food and Agriculture Organization (FAO)

2008. Propuestas de nuevos trabajos para normas del CODEX sobre el chile fresco y el ajo. In: $14^{a}$ Reunión del comité del CODEX sobre frutas y hortalizas frescas. México, D.F. 16-18: 8.

IFA.

2002. Los fertilizantes y su uso. Una guía de bolsillo para los oficiales de extensión. Cuarta edición, 77 p.

INEGI.

2000. XII Censo General de Población y Vivienda. Resultados Definitivos. Tabulados Básicos. Jalisco. México, 2001 INEGI. Censo de Población y Vivienda 2010. Disponible en: http://www. inegi.org.mx,. Consultado: 12/jun/2016.

Jaik-Dipp, A.; Tena-Flores, J.A.

1990. Optimización del proceso de tostado de la semilla de alegría (Amaranthus hypochondriacus) y el diseño de un prototipo de tostadora, en El amaranto (Amaranthus spp.), su cultivo y aprovechamiento. México: El Colegio de Posgraduados.

Judd, R.M.

2008. Plant systematics: a phylogenetic approach ( $3^{\mathrm{a}}$ ed.) Sinauer Associates. Sunderland, UK. 620 p.

Mancilla, V.O.R.; Oropeza, M.J.L.; Martínez, M.M. 2009. Evaluación de terrazas de banco para plantaciones forestales comerciales. Ciencia forestal en México, 34(105): 97-116.
Morón, R.A.; Alayón, G.J.A.

2014. Productividad del cultivo de chile jalapeño (Capsicum anuum L.) con manejo orgánico o convencional en Calakmul, Campeche, México. ECOSUR-Unidad Campeche. 18(3): 35-40.

Muñante, D.D.

2002. Manual de formulación y evaluación de proyectos. $\mathrm{UACH}, \mathrm{Mex}$.

Pedroza, S.A.; Samaniego, G.J.A.

2003. Efecto del subsuelo, materia orgánica y diferentes variedades en el sistema del frijol (Phaseolus vulgaris L.). Rev. Mex. Fitopat., 21: 272-277.

Peña, U.L.S.; Rebollar, R.S.; Callejas, J.N.; Hernández, M.J.; Gómez, T.G.

2015. Análisis de viabilidad económica para la producción comercial de aguacate Hass. Revista Mexicana de Agronegocios. XIX, (36): 1325-1338.

Pérez-Grajales, M.

1997. Mejoramiento genético de hortalizas. Universidad Autónoma Chapingo, 380 p.

Retes, L.R.; Moreno, M.S.; Denogean, B.F.G.; Martín, R.M.; Ibarra, F.F.

2013. Determinación de rentabilidad de trigo en la costa de Hermosillo, Sonora. Sexta Época, 32: 348-357.

Sánchez, E.M.; Espitia Rangel, E.; Osada, K.S.

1991. Etiología de la mancha negra del tallo (Macrophoma sp.) en el amaranto (Amaranthus sp.). I Congreso Internacional del Amaranto. Oaxtepec, Morelos.

Sánchez, M.A.

1980. Potencial agroindustrial del amaranto. Centro de Estudios Económicos y Sociales del Tercer Mundo. México. $238 \mathrm{p}$.

Sánchez, D.G.; Salazar, E.

1994. Rendimiento de maíz forrajero en respuesta a fertilización nitrogenada y densidad de población. Revista Fitotecnia Mexicana. 29: 97-101.

Seufert, V.; Ramankutty, N.; Foley, J.A.

2012. Comparing the yields of organic and conventional agriculture. Nature 485: 229-231.

SIAP.

2010. Un panorama del cultivo del chile. SIAP. México. 20 p.

Tobar, $\mathrm{P}$

2005. Evolución de la rentabilidad de la agricultura y del precio de la tierra, periodo 1983-2002. Panorama socioeconómico, $110 \mathrm{p}$.

Vega, V.D.D.; Ramírez, M.P.

2004. Situación perspectivas del maíz en México. Universidad Autónoma Chapingo. Santiago, Chile. 56 p.

Vivanco, M.

2005. Muestreos estadísticos, diseño y aplicaciones, Chile. Editorial Universitaria. Santiago, Chile. 212 p.

Vijaya, R.

2014. Benefit - cost analysis, Natural Resource Economics, University of Colorado at Boulder. $26 \mathrm{p}$.

Willer, H.; Yussefi, M.

2000. Organic Agriculture Worldwide. INFOAM. Bad Dürkheim, Alemania. 99 p. 